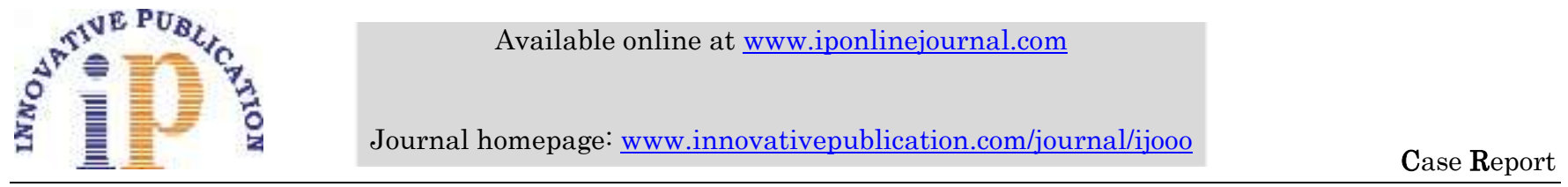

\title{
Bilateral Choroidal Osteoma - A rare entity
}

\author{
Teena Agrawal ${ }^{1 *}$, Mahesh Agrawal ${ }^{2}$ \\ ${ }^{1}$ Consultant, ${ }^{\mathbf{2}}$ Cataract Refractive Surgeon, Dept. of Retina, ${ }^{1}$ Centre For Sight Eye Hospital, Indore, ${ }^{2}$ Shree Ganesh Netralaya - Eye \\ Hospital, Indore, Madhya Pradesh, India
}

\begin{abstract}
A 24 years old female presented to us with the chief complaint of dimness of vision in her right eye since few days and loss of vision in right eye since childhood. On examination she was having well defined yellow to orange slight elevated lesion at macula and peripapillary area and in the left eye marked depigmentation of macula with subretinal fibrosis. She had undergone Optical coherence tomography (OCT) and Ultrasonography (USG-Bscan) of both the eyes. On the basis of clinical findings and investigation, diagnosis of choroidal osteoma was made. The purpose of reporting this case here is that bilateral choroidal osteoma is a very rare entity and whenever a young female is presenting with this kind of lesion in retina, we should keep the possibility of choroidal osteoma in differential diagnosis.
\end{abstract}

Keywords: Choroidal osteoma, Choroidal tumors, Choroidal neovasular membrane, Antivascular endothelial growth factor.

\section{Introduction}

Choroidal osteomas are benign, juxta-papillary tumors that do not require any kind of treatment. They occur mostly in young females (90\%) and are unilateral $(75 \%)^{1}$ Direct ophthalmoscopy, OCT, fundus fluorescein angiography, Indocyanine angiography, USG, X-ray, CT scan etc are used to make the diagnosis. Majority of patients with choroidal osteoma maintain good vision. The probability of loss of visual acuity (20/200 or worse) is more than $50 \%$ by 10 years in these patients, as reported by Shield's et al ${ }^{2,3}$ CNVM is one of the major cause of vision loss noted in these patients. Occurrence of subretinal fluid in the absence of CNVM is also found in few case reports. Anti VEGF intravitreal injections were used successfully in those cases.

\section{Case Report}

A 24 years old female presented to us with the chief complaint of dimness of vision in her right eye since few days. Her left eye was having low vision since childhood. Best corrected visual acuity was recorded 6/18, N8 in the $\mathrm{RE}$ and hand movements in the LE. Slit lamp examination and applanation tonometry was found to be normal in both the eye. Fundus examination showed a well defined yellowish orange, slightly elevated lesion at the macula and along the superior and inferior arcade as shown in (Fig. in1a). Fovea appears spared. There was no haemorrhage or CNVM noted near the edges of lesion. LE fundus showed diffuse retinal pigmentary atrophic changes at the macula and peripapillary area with signs of subretinal fibrosis at the macula and disc gliosis as seen in (Fig. 1b). OCT of RE showing elevated retino-choroidal layer along with presence of subretinal fluid (Fig. 2a). OCT of LE shows irregular elevation of choroid with thinning of all the retinal layers (Fig. 2b). On USG (B scan) we noted the high reflective multiple choroidal lesion persisting on low gain with acoustic shadowing in the RE (Fig. 3). The same was noticed in the left eye also with irregular thickening of choroid with hyper reflectivity. On the basis of clinical findings, OCT and USG test diagnosis of choroidal osteoma is made. Fundus fluorescein angiography was deferred as patient was not willing for any interventional procedure.

In view of SRF overlying the osteoma in the right eye, she was advised intravitreal injection Ranibizumab $(0.5 \mathrm{mg}$ in $0.05 \mathrm{ml}$ ) and the same was given once. On follow-up SRF persisted so we preferred to watch. Patient was continued in follow up for two years with same size of lesion, persistent SRF on OCT and maintained same visual acuity.

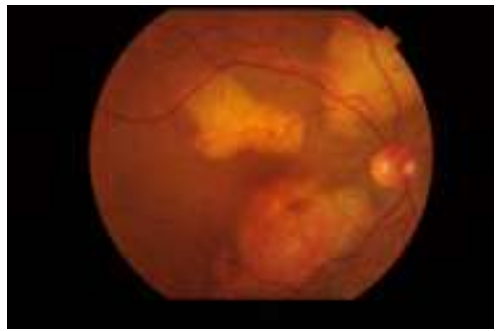

Fig. 1a: Fundus photo RE showing a distinguished yellowto-orange lesion in the macula and along the arcade

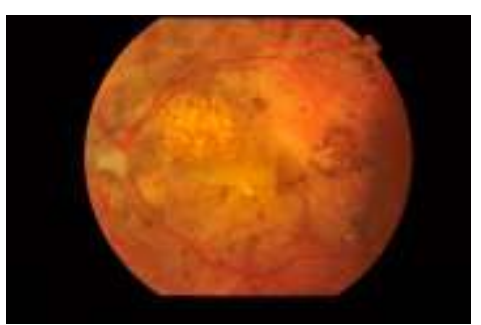

Fig. 1b: Fundus photo LE showing extensive retinal pigment epithelium atrophy at macula and peripapillary area along with subretinal fibrosis at macula and disc gliosis

\footnotetext{
*Corresponding Author: Teena Agrawal, Dept. of Retina, Centre For Sight Eye Hospital, Indore, Madhya Pradesh, India Email: g_teena@hotmail.com http://doi.org/10.18231/j.ijooo.2019.039
} 


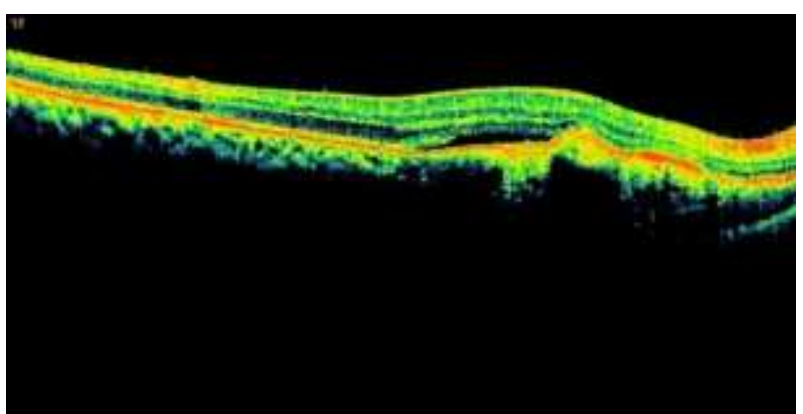

Fig 2a: OCT RE showing SRF overlying the elevated choroid

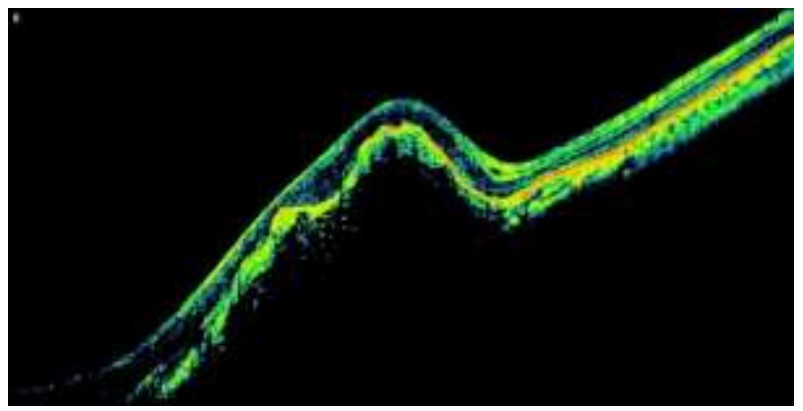

Fig 2b: OCT LE showing irregular elevation of choroid with thinning of retinal layers

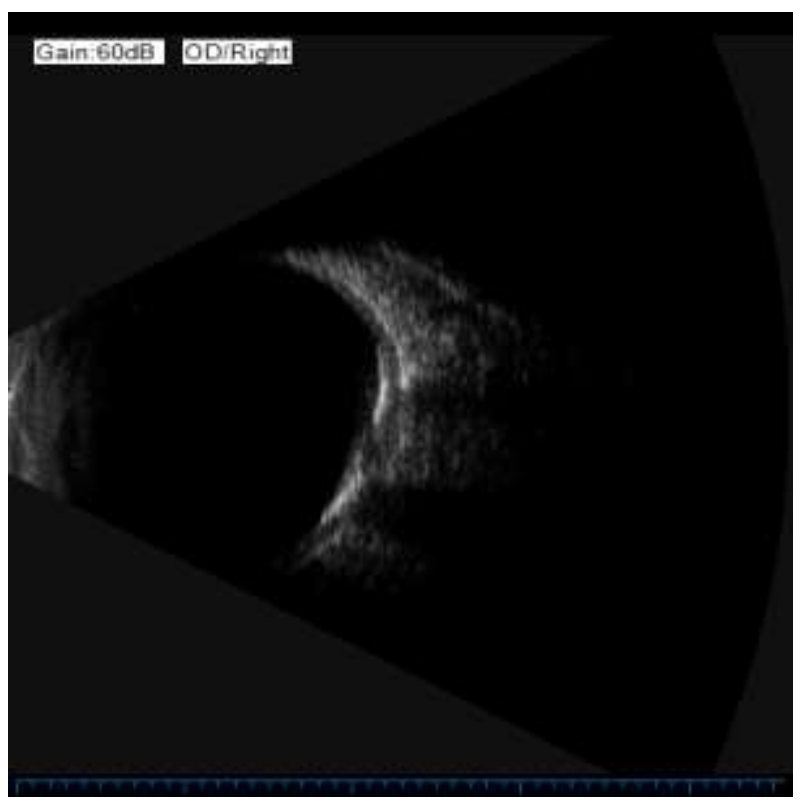

Fig. 3: USG B-scan RE showing hyperreflectivity of the retino-choroid layer with after shadowing

Choroidal osteoma is a rare condition. The largest case series in the literature includes 61 patients over a 26 yearspan of ocular oncology practice in a major tertiary care centre. ${ }^{1}$ Its precise frequency remains unknown. It occurs in all races but predominantly affects young adults in their early twenties with a large range from few months old to late sixties. It has a predilection for women and is unilateral in around 80 percent of cases. ${ }^{1,2,3}$ The exact etiology of choroidal osteoma is unknown. There has been found no association between choroidal osteoma and any abnormality in blood chemistries such as calcium, phosphorus, alkaline phosphatase, complete blood count and urinalysis. ${ }^{3}$ Choroidal osteoma appear as a deep, orange-yellow lesion with distinct geographic or scalloped borders and branching vessels on the surface of the tumor. The lesion colour relates to the level of retinal pigment epithelium (RPE) depigmentation. In early stages choroidal osteomas tend to have an orange-red colour, whereas in later stages they have a yellowish tint due to RPE depigmentation ${ }^{4}$. Histopathology illustrates dense bony trabeculae with marrow spaces traversed by pathognomonic dilated thinwalled blood vessels, termed spider or feeder vessels. ${ }^{5}$ Tumor decalcification and resolution, originally described by Trimble ${ }^{6}$ in 1988 occurs in around 50 percent of case and is associated with long term poor visual acuity as seen in our patient in LE.

Choroidal osteomas are included in the large differential diagnosis of ocular tumors and ocular calcification. These include amelanotic choroidal melanoma, amelanotic choroidal nevus, metastatic choroidal carcinoma, circumscribed choroidal hemangioma, disciform macular degeneration, posterior scleritis, idiopathic sclerochoroidal calcification, choroidal cartilage, leukemia and retinoblastoma.

Choroidal osteomas are also known to be associated with subretinal fluid, hemorrhages and serous retinal detachment. Serous retinal detachment occurs in choroidal osteoma frequently in the absence of CNV. It is speculated to be the result of multiple pinpoint RPE leakage sites over the osteoma as detected by fluorescein angiography. Alternatively, atrophy of the RPE and Bruch membrane is thought to decrease their capacity to remove subretinal fluid emanating from the disrupted outer blood-retinal barrier. In our case we noticed the same thing in the RE i.e. presence of SRF on OCT without any CNVM.

There is no standard protocol for treatment for choroidal osteomas, but therapies are directed for complications arising from choroidal neovascularization (CNV) and subretinal fluid. Focal Laser, Transpupillary therapy, photodynamic therapy and intravitreal anti VEGF injections are tried with variable success rate. We also used intravitreal anti VEGF injection once in the RE but the amount of SRF we noticed before giving injection was found to be the same even after giving injection in contrast to earlier reports ${ }^{6}$ We decided to watch this case and she maintained the same visual acuity in 2 year follow up.

\section{Abbreviations}

Optical coherence tomography (OCT), ultrasound (USG), choroidal neovasular membrane (CNVM), Retinal pigment epithelium (RPE), CT scan(computed tomography), right eye(RE), left eye (LE), subretinal fluid(SRF), vascular endothelial growth factor(VEGF)

\section{Source of Funding}

None. 


\section{Conflict of Interest}

None.

\section{References}

1. Shields CL, Sun H, Demirci H, Shields JA. Factors predictive of tumor growth, tumor decalcification, choroidal neovascularization, and visual outcome in 74 eyes with choroidal osteoma. Arch Ophthalmol 2005;123:1658-66.

2. Aylward GW, Chang TS, Pautler SE, Gass JD. A long-term follow-up of choroidal osteoma. Arch Ophthalmol 1998;116:1337-41.

3. Shields CL, Shields JA, Augsburger JJ. Choroidal osteoma. Surv Ophthalmol 1988;33:17-27.
4. Chen J, Lee L, Gass JD. Choroidal osteoma: Evidence of progression and decalcification over 20 years. Clin Exp Optom 2006;89:90-4.

5. Gass JD, Guerry RK, Jack RL, Harris G. Choroidal osteoma. Arch Ophthalmol 1978;96:428-35.

6. Song JH, Bae JH, Rho MI, Lee SC. Intravitreal bevacizumab in the management of subretinal fluid associated with choroidal osteoma. Retina 2010;30:945-51.

How to cite this article: Agrawal T, Agrawal M, Bilateral Choroidal Osteoma - A rare entity, Int $J$ Ocul Oncol Oculoplasty 2019;5(3):150-2. 